\section{Case Reports in Ophthalmology}

\title{
Surgical Management of a Patient with Anterior Megalophthalmos, Lens Subluxation, and a High Risk of Retinal Detachment
}

\author{
María Carmen Guixeres Esteve Augusto Octavio Pardo Saiz \\ Lucía Martínez-Costa Samuel González-Ocampo Dorta \\ Pedro Sanz Solana \\ Department of Ophthalmology, Hospital Dr. Peset, Valencia, Spain
}

\section{Keywords}

Anterior megalophthalmos · Lens subluxation · Vitrectomy · Retinal detachment

\section{Abstract}

The early development of lens opacities and lens subluxation are the most common causes of vision loss in patients with anterior megalophthalmos (AM). Cataract surgery in such patients is challenging, however, because of anatomical abnormalities. Intraocular lens dislocation is the most common postoperative complication. Patients with AM also seem to be affected by a type of vitreoretinopathy that predisposes them to retinal detachment. We here present the case of a 36-year-old man with bilateral AM misdiagnosed as simple megalocornea. He had a history of amaurosis in the right eye due to retinal detachment. He presented with vision loss in the left eye due to lens subluxation. Following the removal of the subluxated lens, it was deemed necessary to perform a vitrectomy in order to prevent retinal detachment. Seven months after surgery, an Artisan ${ }^{\circledR}$ Aphakia iris-claw lens was implanted in the anterior chamber. Fifteen months of follow-up data are provided. 


\section{Introduction}

Anterior megalophthalmos (AM) is a rare, inherited, usually symmetrical, developmental anomaly of the anterior segment of the eye. It has a constellation of clinical signs including megalocornea, deep anterior chamber, posterior positioning of the iris and lens, ciliary ring enlargement, myopia, miosis, pupil displacement, noncongenital glaucoma, a large capsular bag, lens subluxation, early cataract, pigment dispersion syndrome, a vitreous index of $<69 \%$, and vitreoretinopathy predisposing to retinal detachment. Differentiating between $\mathrm{AM}$ and primary congenital glaucoma is very important [1].

AM commonly involves an X-linked recessive inheritance pattern [1, 2]. However, all modes of inheritance have been reported.

There have been some case reports of AM since 1931, most of them describing different techniques of cataract surgery and intraocular lens (IOL) implantation. We here present, to our knowledge, the first patient with AM to receive an anterior chamber iris-claw IOL in association with pars plana lensectomy and vitrectomy.

\section{Case Report}

A 36-year-old man presented at our hospital having experienced reduced vision in the left eye for 3 days. His ocular medical background included a diagnosis of bilateral simple megalocornea, myopia, and cataract. Eleven years ago, he received treatment at another hospital for retinal detachment in the right eye; he has low vision in that eye since that time. His best corrected visual acuity was OD amaurosis and OS 0.2. The refractive error was OD $-14.50 /-3.75 \times 175^{\circ}$, and OS $-4.25 /-1.25 \times 66^{\circ}$. The corneas were clear and measured OD $14.05 \mathrm{~mm}$ and OS $13.90 \mathrm{~mm}$ horizontally, as determined using a Visante ocular coherence tomography (OCT) device (Carl Zeiss Meditec, Germany) (Fig. 1).

Slit-lamp examination revealed bilateral hyper-deep anterior chambers, posterior subcapsular opacity (Fig. 2), anterior corneal embryotoxon, miosis, nasal pupil displacement, Krukenberg's spindle, iris transillumination defects, iridodonesis, phakodonesis in the left eye, heavy angle pigmentation, and temporal and inferior subluxation of the lens in the left eye (Fig. 2) with vitreous herniation at the top. The intraocular pressure, measured using an applanation tonometer, was OD $13 \mathrm{~mm} \mathrm{Hg}$ and OS $15 \mathrm{~mm} \mathrm{Hg}$. The fundus and the macular OCT (Cirrus, Carl Zeiss Meditec) were unremarkable in the OS (Fig. 3). Central corneal thickness, determined using a Visante OCT, was $450 \mu \mathrm{m}$ in both eyes (Fig. 1). Using an IOL Master 500 noncontact optical biometer (Carl Zeiss Meditec), and employing the SRK/T formula, an OS IOL power of 12.40 diopters (with an A-constant of 119) was determined. Examination with a 3000P specular microscope (TOPCON, Japan) returned endothelial counts of 1,839 cells $/ \mathrm{mm}^{2}(\mathrm{OS})$. The anterior chamber depths, determined using a Visante OCT, were OD $5.32 \mathrm{~mm}$ and OS $5.61 \mathrm{~mm}$ (Fig. 1). Ocular axial length values, measured using the same IOL Master 500 apparatus as above, were OD $24.27 \mathrm{~mm}$ and OS $25.51 \mathrm{~mm}$.

Systemic examination was normal. No relevant family history was detected. Surgical management consisted of an uneventful pars plana lensectomy and vitrectomy. No IOL was implanted intraoperatively. Seven months after surgery, an 11-diopter Artisan ${ }^{\circledR}$ Aphakia iris-claw lens (Ophtec B.V., The Netherlands) with an A-constant of 115.7 was implanted in the anterior chamber (Fig. 4). Upon the last examination made, the refractive error was $+0.25 /-0.50 \times 140^{\circ}(\mathrm{OS})$ and the best-corrected visual acuity was 0.8 . 


\section{Discussion}

This report describes the management of a patient with AM showing lens subluxation and anterior chamber vitreous prolapse in the left eye. The patient had suffered retinal detachment in the right eye some years before. This was unsuccessfully treated, resulting in a poor functional result. Ahmadieh et al. [3] reported the most common abnormalities of the fundus. Some of them were vitreous degeneration, areas of lattice degeneration, spontaneous vitreous hemorrhage, peripheral retinal neovascularization, and retinal detachment.

Fundus examinations are necessary, and prophylactic procedures should be followed if required. The retina of the present patient was examined at each visit. A pars plana lensectomy and vitrectomy were deemed necessary since this procedure permitted lens removal within a closed ocular system, thereby limiting vitreal traction and preventing retinal detachment [4]. It was preferred over phacoemulsification and anterior vitrectomy. Javadi et al. [5] reported 6 cases of cataract extraction in eyes with AM. In 1 patient, vitreous loss occurred during surgery, and anterior vitrectomy was performed; 3 months later, the patient suffered retinal detachment. In addition, vitrectomy allowed a good intraoperative overview of vitreal and retinal structures, giving the possibility of repairing an unstable retina if it had been revealed during surgery [4].

IOL dislocation is the most frequent postoperative complication [2]. Different strategies have been used to evade it. A standard posterior chamber IOL is likely to decenter $[2,5,6]$; options include an iris-claw IOL [7-10], an iris-sutured IOL [6, 11, 12], or a large custommade posterior chamber IOL $[11,13]$. It has been reported that iris-sutured IOLs can eventually become loose in eyes with AM [9]. Aphakia iris-claw lenses, e.g., Artisan ${ }^{\circledR}$ (Ophtec BV) or Verisyse ${ }^{\circledR}$ (Abbott Medical Optics Inc., Santa Ana, CA, USA), were first used in patients with AM in 2006, implanting them in the anterior $[2,7,9]$ or posterior chambers $[8,10]$. Satisfactory results were achieved, although in 1 eye refixation was required [7]. To our knowledge, the present patient is the fifth with AM to receive an anterior chamber iris-claw IOL and the first to receive such a lens in association with pars plana lensectomy and vitrectomy $[2,7,9]$. These lenses seem to be an excellent choice for AM patients; the way the lens fixes to the iris provides stability to the IOL despite the abnormal anatomy encountered.

Visual rehabilitation was successful. Even if the patient showed pseudophakodonesis, he experienced no image shift. Possible complications, such as IOL subluxation, elevated intraocular pressure, cystoid macular edema, or postoperative hyperopia, did not occur $[7,14$, 15]. Hyperopia can appear because of the increased anterior chamber depth of the eye [7, 15]. To prevent this, some authors [15] recommend trying to achieve a degree of myopic refraction. This advice proved successful with the present patient, whose postoperative refractive error was close to emmetropia.

In conclusion, lensectomy and pars plana vitrectomy, with the implantation of an Artisan ${ }^{\circledR}$ IOL, proved to be a good option for this patient with AM, lens subluxation, and a high risk of retinal detachment. After 15 months of follow-up, the results remain satisfactory; the patient has very good visual acuity and no retinal complications.

\section{Acknowledgements}

We thank the foundation Fundación para el Fomento de la Investigación Sanitaria y Biomédica (FISABIO) for providing financial support for the publication fee. 


\section{Statement of Ethics}

The patient has given his informed consent.

\section{Disclosure Statement}

The authors declare no conflicts of interest.

\section{References}

1 American Academy of Ophthalmology: Basic and Clinical Sciences Course. Sec 8. External Disease and Cornea. San Francisco, CA, American Academy of Ophthalmology, 2011-2012, pp 253-254.

2 Wang Q, Xu W, Zhu Y, Li J, Zhang L, Yao K: Misdiagnosis induced intraocular lens dislocation in anterior megalophthalmos. Chin Med J 2012;125:3180-3182.

3 Ahmadieh H, Banaee T, Javadi MA, Jafarinasab MR, Yazdani S, Sajjadi H: Vitreoretinal disorders in anterior megalophthalmos. Jpn J Ophthalmol 2006;50:515-523.

-4 Kodjikian L, Beby F, Spire M, Gambrelle J, Hubert I, Burillon C, Grange JD, Garweg JG: Combined pars plana phacofragmentation, vitrectomy, and Artisan lens implantation for traumatic subluxated cataracts. Retina 2006;26:909-916.

-5 Javadi MA, Jafarinasab MR, Mirdehghan SA: Cataract surgery and intraocular lens implantation in anterior megalophthalmos. J Cataract Refract Surg 2000;26:1687-1690.

-6 Li Y, Liu F, Zhang Q, Xiong Y: Cataract surgery and intraocular lens implantation in anterior megalophthalmos. Int J Ophthalmol 2012;5:648-649.

7 Oetting TA, Newsom TH: Bilateral Artisan lens for aphakia and megalocornea: long-term follow-up. J Cataract Refract Surg 2006;32:526-528.

-8 Lee GA, Hann JV, Braga-Mele R: Phacoemulsification in anterior megalophthalmos. J Cataract Refract Surg 2006;32:1081-1084.

9 Welder J, Oetting TA: Megalocornea. EyeRounds.org. September 17, 2010. . http://www.EyeRounds.org/cases/121-megalocornea.htm (accessed September 13, 2014).

10 Galvis V, Tello A, Miotto G, Rangel CM: Artisan aphakic lens for cataract surgery in anterior megalophthalmos. Case Rep Ophthalmol 2012;3:428-433.

11 Sharan S, Billson FA: Anterior megalophthalmos in a family with 3 female siblings. J Cataract Refract Surg 2005;31:1433-1436.

12 Dua HS, Azuara-Blanco A, Pillai CT: Cataract extraction and intraocular lens implantation in anterior megalophthalmos. J Cataract Refract Surg 1999;25:716-719.

13 Marques Vaz F, Osher RH: Cataract surgery and anterior megalophthalmos: custom intraocular lens and special considerations. J Cataract Refract Surg 2007;33:2147-2150.

14 De Silva SR, Arun K, Anandan M, Glover N, Patel CK, Rosen P: Iris-claw intraocular lenses to correct aphakia in the absence of capsule support. J Cataract Refract Surg 2011;37:1667-1672.

15 Assia EI, Segev F, Michaeli A: Cataract surgery in megalocornea. Comparison of 2 surgical approaches in a single patient. J Cataract Refract Surg 2009;35:2042-2046. 


\section{Case Reports in Ophthalmology}

\begin{tabular}{l|l}
\hline Case Rep Ophthalmol 2017;8:61-66 \\
\hline DOI: 10.1159/000456068 & $\begin{array}{l}\text { @ 2017 The Author(s). Published by S. Karger AG, Basel } \\
\text { www.karger.com/cop }\end{array}$ \\
\hline
\end{tabular}

Guixeres Esteve et al. Surgical Management of a Patient with Anterior Megalophthalmos, Lens Subluxation, and a High Risk of Retinal Detachment

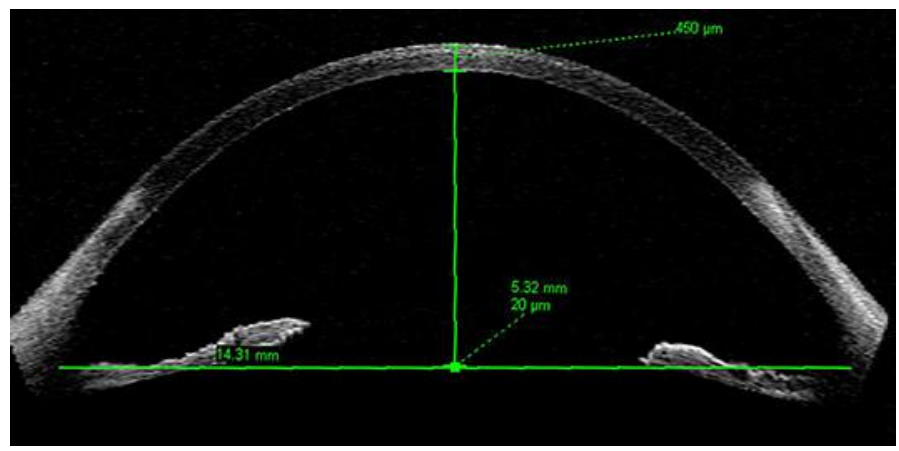

Fig. 1. Visante OCT-acquired image of the left eye, showing thin corneas, deep anterior chambers, and megalocornea.

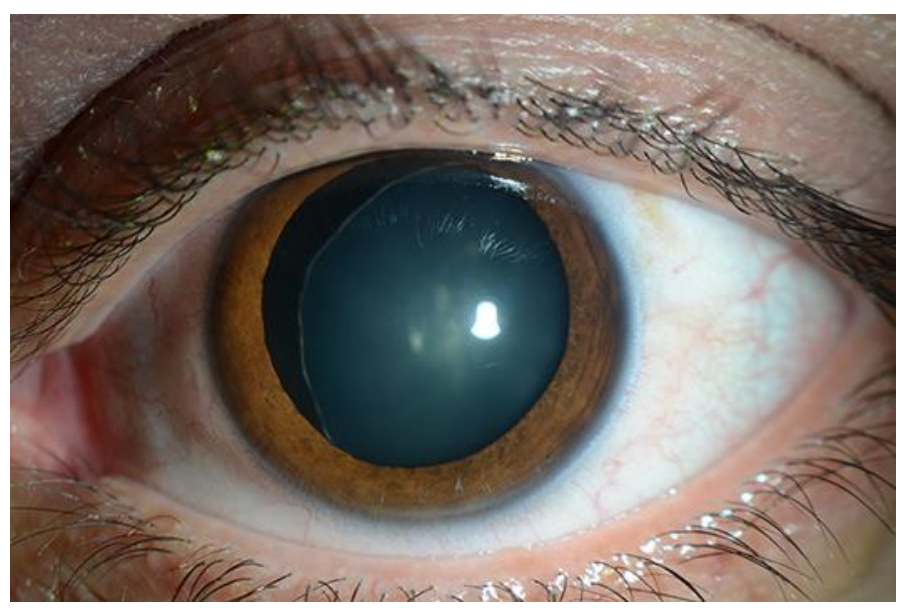

Fig. 2. Frontal view of the left eye with anterior megalophthalmos, showing megalocornea, lens subluxation, posterior subcapsular opacity, and anterior corneal embryotoxon.

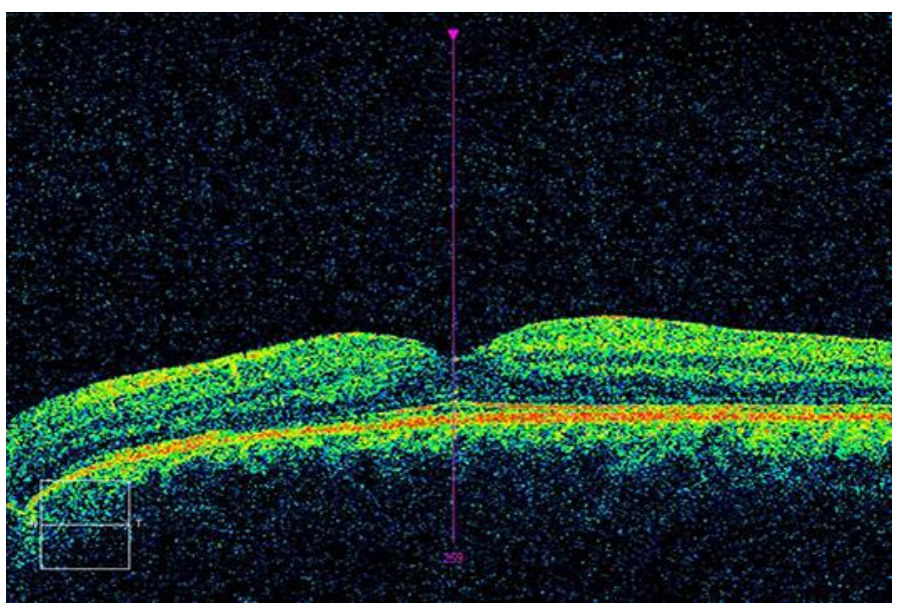

Fig. 3. Normal macular ocular coherence tomography of the left eye. 


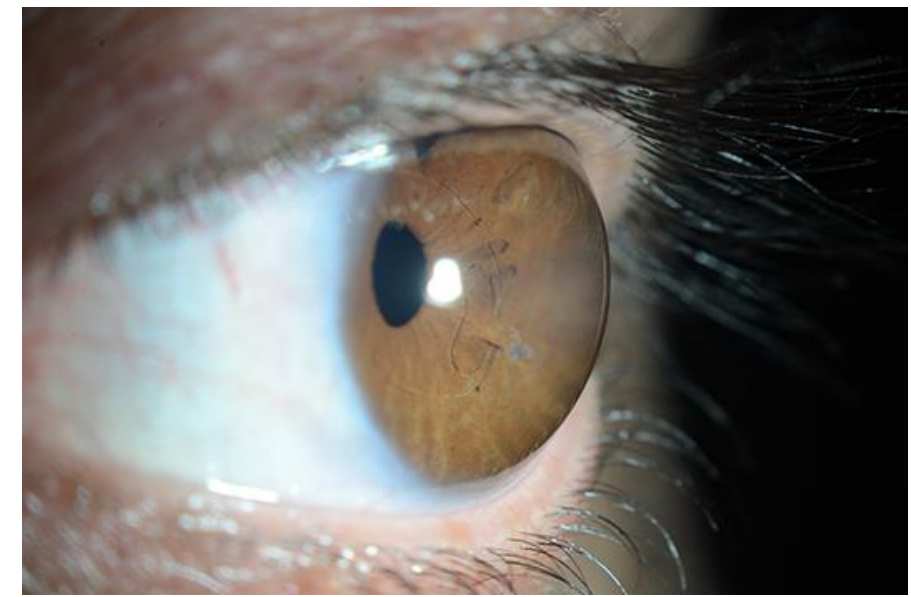

Fig. 4. Postoperative view of the left eye showing the hyper-deep chamber and the anterior chamber Arti$\operatorname{san}{ }^{\circledR}$ intraocular lens in situ at 7 months. 